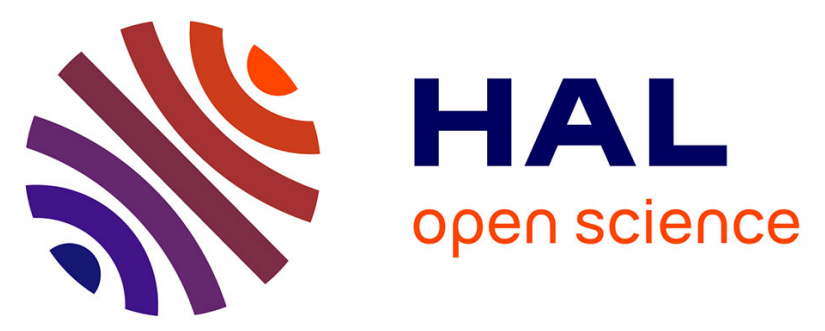

\title{
Charge-Transfer versus Charge-Separated Triplet Excited States of [Re I (dmp)(CO) 3 (His124)(Trp122)] + in Water and in Modified Pseudomonas aeruginosa Azurin Protein
}

\author{
Marco Marazzi, Hugo Gattuso, Maria Fumanal, Chantal Daniel, Antonio
}

Monari

\section{To cite this version:}

Marco Marazzi, Hugo Gattuso, Maria Fumanal, Chantal Daniel, Antonio Monari. Charge-Transfer versus Charge-Separated Triplet Excited States of [Re I (dmp)(CO) 3 (His124)(Trp122)] + in Water and in Modified Pseudomonas aeruginosa Azurin Protein. Chemistry - A European Journal, 2019, 25 (10), pp.2519-2526. 10.1002/chem.201803685 . hal-03037929

\author{
HAL Id: hal-03037929 \\ https://hal.science/hal-03037929
}

Submitted on 4 Dec 2020

HAL is a multi-disciplinary open access archive for the deposit and dissemination of scientific research documents, whether they are published or not. The documents may come from teaching and research institutions in France or abroad, or from public or private research centers.
L'archive ouverte pluridisciplinaire HAL, est destinée au dépôt et à la diffusion de documents scientifiques de niveau recherche, publiés ou non, émanant des établissements d'enseignement et de recherche français ou étrangers, des laboratoires publics ou privés. 


\title{
Charge transfer vs. charge separated triplet excited states of $\left[\operatorname{Re}^{\prime}(\mathrm{dmp})(\mathrm{CO})_{3}(\mathrm{His} 124)(\operatorname{Trp} 122)\right]$ in water and in modified pseudomonas aeruginosa azurin protein.
}

\author{
M. Marazzi, ${ }^{a, b}$ H. Gattuso, ${ }^{a}$ M. Fumanal, ${ }^{c}$ C. Daniel,,${ }^{c,}$ and A. Monari ${ }^{a,{ }^{*}}$
}

\begin{abstract}
We present a computational investigation of the triplet excited states of a Rhenium complex electronically coupled with a tryptophan side chain and bound to an azurin protein. In particular, using high-level molecular modelling, we evidence how the electronic properties of the excited state manifolds strongly depend on the coupling with the environment. Indeed, only when explicitly taking into account the protein environment, two stable triplet states of metal-to-ligand charge transfer or charge separated nature can be recovered. Their interconversion gives rise to a ns time scale electron-transfer process observed experimentally. In addition, we also demonstrate how the Re complex plus tryptophan system in a water environment, experiences a too high flexibility preventing the two chromophores to be electronically coupled. This occurrence disables the formation of a charge-separated state, rationalizing the experimental observation. Our successful strategy requires a multiscale approach combining molecular dynamics and quantum chemistry. In this context, we also present the strategy used to parameterize the force fields for the electronic triplet states of the metal complex.
\end{abstract}

\section{Introduction}

The excited states properties of isolated metal complexes are the subject of numerous computational investigations based on electronic structure theory and quantum dynamics for the interpretation of their fascinating behavior [1-6]. However only a few pioneering studies have been devoted to the effects of the molecular surroundings on these properties [7-11]. On the other hand, since metal complexes properties are most often deployed in complex environments, including solid state, organized materials, and biosystems, their rationalization is of crucial importance.

Most of the time, solvent effects are modeled implicitly by a simple continuum polarizable medium around a cavity, as the

[a] Dr. M. Marazzi, Dr H. Gattuso, Dr. A. Monari

Université de Lorraine and CNRS, LPCT UMR 7019, Boulevard des Aiguillettes, Vandoeuvre-lès-Nancy, F-54000 Nancy, France antonio.monari@univ-lorraine.fr

[b] Dr. M. Marazzi, Departamento de Química, Centro de Investigacíon en Síntesis Química (CISQ), Universidad de La Rioja, Madre de Dios, 53, 26006 Logroño, Spain

[c] Dr. M. Fumanal, Dr. C. Daniel

Laboratoire de Chimie Quantique, Institut de Chimie Strasbourg, UMR-7177 CNRS/Université de Strasbourg, 1 Rue Blaise Pascal BP 296/R8, F-67008 Strasbourg, France.

c.daniel@unistra.fr

Supporting information for this article is given via a link at the end of the document.
Polarizable Continuum Model (PCM) [12-15] or the Conductorlike Screening Model (COSMO) [16-18]. Even if undoubtedly successful, this approach suffers from severe drawbacks, in particular lacking the flexibility to describe specific effects such as hydrogen bonds that may tune specific photophysical behaviours. Furthermore, the electronic density reorganization leading to charge separation between the metal complex and its biological environment[19-21] is entirely neglected. Those factors can lead to an erroneous description of the excited states' electronic densities and consequently to an incorrect interpretation of specific photophysical or photochemical features. As a paradigmatic example, one can cite the study of the emission properties and absorption spectra of organometallic $\operatorname{Ru}(\mathrm{II}) \mathrm{DNA}$ intercalators $[7,22,23]$ or of a $\operatorname{Re}(\mathrm{I})$ complex probe for DNA-mediated charge transport [10].

In particular, charge separated (CS) excited states are of paramount importance in photo-induced electron transfer processes initiated by visible light absorption for transition metal complexes possessing low-lying metal-to-ligand-charge-transfer (MLCT) excited states. This has been firmly established by the study of mutants of pseudomonas aeruginosa azurin redox protein $[19-21,24]$, sensitized with UV-visible light absorbing Ruor Re-complexes, able to ultimately lead to coherent photoinduced charge transfer to the native copper center over more than $30 \AA$. The charge transfer process is supposed to be initiated by the population of MLCT states localized on the metal complex, followed by charge-separation with the formation of a CS state between the metal complex and a coupled, close-by, tryptophan whose evolution will ultimately lead to a chargetransfer to the copper center. Indeed, time-resolved experiments have put in evidence the formation of $a^{3} \mathrm{CS}$ in the protein by two different channels, namely via a direct ultra-fast decay $(<1 \mathrm{ps})$ from the metal complex chromophore, $\left[\operatorname{Re}^{\prime}(\mathrm{dmp})(\mathrm{CO})_{3}(\mathrm{His} 124)(\operatorname{Trp} 122)\right] \quad(\mathrm{dmp}=4,7$-dimethylphenanthroline), or via a slower process (0.5-1.5 ns) across a ${ }^{3} \mathrm{MLCT} /{ }^{3} \mathrm{CS}$ equilibrium. Furthermore, while the long-range charge-transfer phenomena is found to proceed efficiently when the chromophores are organized by the protein scaffold (Figure 1a), a model system composed of the metal complex covalently bound to the tryptophan residue in water solution (Figure 1b) does not show any evidence of this CS state formation.

This puzzling kinetics calls for a theoretical interpretation, including environment effects, of the elementary steps that lead to ${ }^{3} \mathrm{CS}$, key state for the two-step electron tunnelling in $\operatorname{Re}(\mathrm{I})$ modified azurin [19]. For this purpose it is necessary to develop new methods and specific Force Fields (FF) able to model the lowest triplet states of the $\operatorname{Re}(\mathrm{I})$ complex in various environments and to characterize the presence of both ${ }^{3} \mathrm{MLCT}$ and ${ }^{3} \mathrm{CS}$ in the protein. 

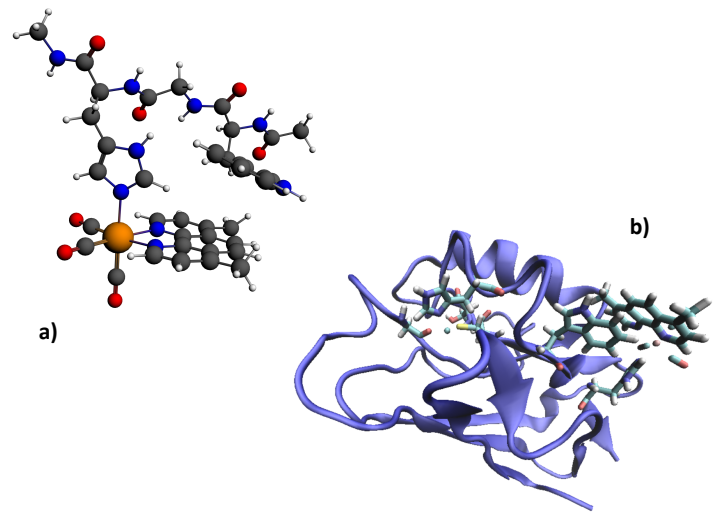

Figure 1. a) Optimized structure of $\left[\operatorname{Re}^{\mathrm{I}}(\mathrm{dmp})(\mathrm{CO})_{3}(\mathrm{His} 124)(\operatorname{Trp} 122)\right]$ model system in water solution and $b$ ) the structure of the functionalized azurin protein, with the Re (right) and $\mathrm{Cu}$ (left) complexes evidenced in licorice representation.

In the present contribution we resort to an extended molecular modelling and simulation of the behaviour of $\left[\operatorname{Re}^{\prime}(\mathrm{dmp})(\mathrm{CO})_{3}(\mathrm{His} 124)(\operatorname{Trp} 122)\right]$ both in water and embedded in the azurin protein. In particular, we will consider the environment effects on the properties of singlet and triplet excited states ensembles, proving the crucial role of the protein environment in providing a stable CS state exploiting hybrid quantum mechanics/molecular mechanics (QM/MM) frameworks [25]. In addition, we also investigated the role of the environment in shaping the conformational space of both the ground and the triplet states manifold by using classical molecular dynamics (MD). To this end we also report the explicit parameterization of force fields describing not only the metal complex ground state but also the behavior of the different triplet states.

The objective of this work is on one side to characterize the ${ }^{3} \mathrm{MLCT} /{ }^{3} \mathrm{CS}$ states observed experimentally in the protein, and on the other side to rationalize the lack of charge transfer observed for the model system in water solution. Our results will also constitute the basis for further studies modelling the kinetic of the photoinduced charge transfer process by means of either biased QM/MM molecular dynamics or non-adiabatic molecular dynamics techniques. At the same time, our contribution will allow a better comprehension of the subtle and complex equilibrium involving dynamic and electronic effects in environmentally driven photo-excited phenomena experienced by organometallic compounds.

\section{Results and Discussion}

Electronic structure of $\left[\operatorname{Re}^{\prime}(d m p)(C O)_{3}(H i s 124)(\operatorname{Trp} 122)\right]$ in water

The optimized structure of the singlet ground state of $\left[\operatorname{Re}^{\prime}(\mathrm{dmp})(\mathrm{CO})_{3}(\mathrm{His} 124)(\operatorname{Trp} 122)\right]$ in water is depicted in Figure $1 \mathrm{a}$, whereas the optimized structures of the low-lying triplet excited states ${ }^{3} \mathrm{MLCT}$ and ${ }^{3} \mathrm{CS}$ are represented in Figure 2 together with their spin densities. Without geometrical constraints the ${ }^{3} \mathrm{MLCT}$ optimization converges to the ${ }^{3} \mathrm{CS}$. It is worth noting that only when using different functionals, namely PBE0 [26] for the ${ }^{3} \mathrm{CT}$ state and M06 [27] for the ${ }^{3} \mathrm{CS}$ state with
$\mathrm{PCM}-\mathrm{H}_{2} \mathrm{O}$ solvent model, the two structures have been obtained without any constraint by Blanco-Rodriguez et al. [19]. This illustrates the limit of a static approach when investigating the structural properties of excited states in transition metal complexes with flexible ligands.

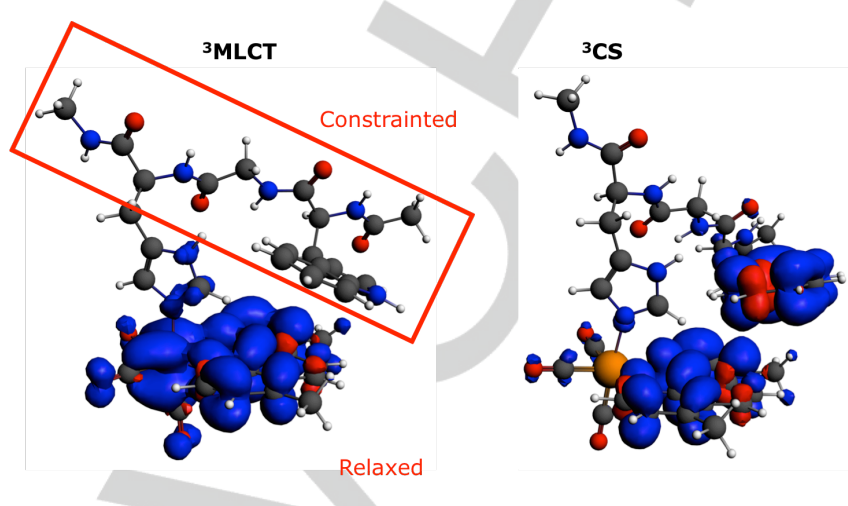

Spin Re: $0.4 \quad \Delta \mathrm{E}<2.0 \mathrm{kcal} \mathrm{mol}^{-1} \quad$ Spin Re: 0.0

Figure 2. Spin densities of ${ }^{3} \mathrm{MLCT}$ (left) and ${ }^{3} \mathrm{CS}$ (right) excited states optimized structures of $\left[\operatorname{Re}^{\prime}(\mathrm{dmp})(\mathrm{CO})_{3}(\mathrm{His} 124)(\operatorname{Trp} 122)\right]$ in water (COSMO model).

The low-energy absorption spectrum is characterized by six CS states, namely three singlets of modest oscillator strengths $(\sim$ $10^{-3}$ ) calculated at $2.80,3.0$ and $3.36 \mathrm{eV}$ and three triplet calculated at $2.79,2.98$ and $3.36 \mathrm{eV}$. Two ${ }^{1} \mathrm{MLCT}$ states absorb at $3.26 \mathrm{eV}$ and $3.43 \mathrm{eV}$ with oscillator strengths of $8.010^{-3}$ and $3.010^{-2}$, respectively. Low-lying ${ }^{3} \mathrm{MLCT}$ range between 3.04 and $3.22 \mathrm{eV}$. The complete electronic structure data including frontier molecular orbitals and one-electron contributions of the lowest excited states is collected in Table S1 of SI. The experimental evidence of the appearance of a ${ }^{3} \mathrm{CS}$ state in less than 1 ps [19] could be explained by an ultrafast decay from the ${ }^{1}$ MLCT absorbing states of $\left[\operatorname{Re}^{\prime}(\mathrm{dmp})(\mathrm{CO})_{3}(\mathrm{His} 124)(\operatorname{Trp} 122)\right]$ to the lowest ${ }^{3} \mathrm{CS}$ state calculated at $2.79 \mathrm{eV}$.
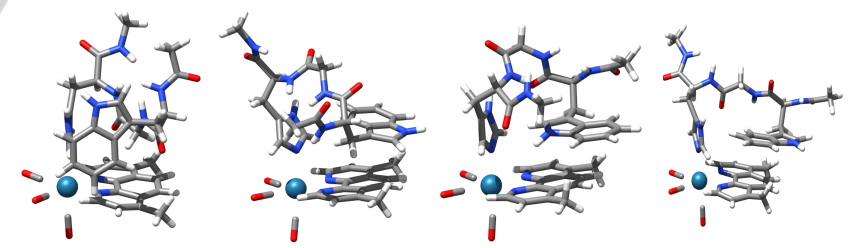

Figure 3. Principal conformations extracted from a clustering analysis of the GS dynamics of $\left[\operatorname{Re}^{\prime}(\mathrm{dmp})(\mathrm{CO})_{3}(\mathrm{His} 124)(\operatorname{Trp} 122)\right]$ in water.

$M D$ of $\left[\operatorname{Re}^{\prime}(d m p)(C O)_{3}(\right.$ His 124)(Trp122)] in water and embedded in azurin.

In order to go beyond a static description of the different excited states we also analyse the GS MD of the $\left[\operatorname{Re}^{\prime}(\mathrm{dmp})(\mathrm{CO})_{3}(\mathrm{His} 124)(\operatorname{Trp} 122)\right]$ in water solution. It clearly turns out (Figure 3 ) that the organometallic complex exhibits a great flexibility around the peptide bond bridging the tryptophan side chain to the imidazole unit. As a consequence, one can clearly see that the coupling between $\mathrm{dmp}$ and the tryptophan chromophore is globally lost with the two subunits presenting relative arrangements far from the QM optimized ones. Most 
notably, the same coupling loss between the chromophores is also evident in the case of both ${ }^{3} \mathrm{MLCT}$ and ${ }^{3} \mathrm{CS}$ state, as can be inferred from the tryptophan-dmp distance reported in Figure 4 for the three electronic states, with a distance between $\mathrm{dmp}$ and the tryptophan moiety reaching up to $18 \AA$. Moreover a highly oscillating behavior is observed, with only very few stable conformations populated for very limited amounts of time during the MD simulation. a)

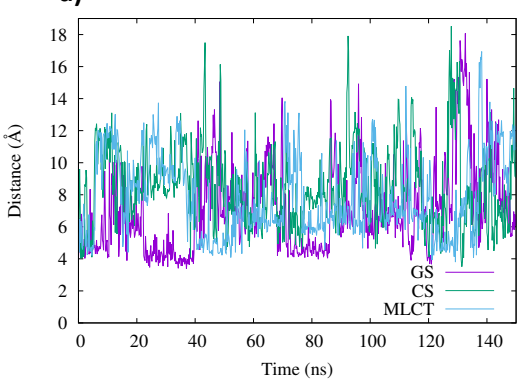

b)

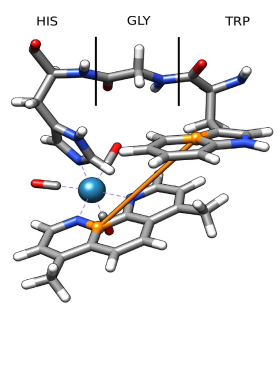

Figure 4. a) Time evolution of the dmp-tryptophan distance over the MDs of the complex in water solution; b) Explicit definition of the atoms used to calculate the distances (orange).

The important flexibility and the consequent loss of the stacking between $\mathrm{dmp}$ and tryptophan $\pi$-conjugated systems bring however important photophysical consequences. Indeed, the absence of a favorable coupling can certainly lead to the hampering of the electron-transfer process that has been observed in the protein. Indeed, in a Marcus like picture nonstacked chromophores can be related to both an increase of the kinetic activation energy required and to a decrease in the reorganization energy, all in all leading to a drop on the transfer kinetic constant. Hence, the absence of an organized structure in this model system easily explains that electron-transfer is not observed experimentally.

The picture is instead greatly different when the conformational space of the chromophore is constrained by the protein environment. Indeed, in this case (Figure 5) the dmp-tryptophan distance is relatively constant all along the MD simulation for the GS, showing only very limited and punctual oscillations and peaking at around $7 \AA$. Similarly, a more constrained evolution compared to the water solution case, is observed for the two triplet states for which the distance between the two conjugated rings stays relatively short, even if an increase can be observed for ${ }^{3} \mathrm{MLCT}$ after $150 \mathrm{~ns}$ of simulation. Note that the ${ }^{3} \mathrm{CS}$ state necessitates a longer time to equilibrate and experiences a general much higher flexibility.

A deeper insight on the structural properties of the excited states can be seen from the dominant configurations obtained from a clustering analysis on the three states, as reported in Figure 5. Indeed, in the case of the GS one can observe an almost ideal stacking of the dmp and thryptophan side chain that can also be easily correlated to the very marginal oscillation of the distance distribution. The behavior of the triplet states is however less intuitive and presents some unexpected characteristic worth of analysis. Indeed, in the case of the ${ }^{3} \mathrm{MLCT}$ state, one can observe a rotation of the peptide bond bridging the tryptophan and its displacement towards the $(\mathrm{CO})_{3}$ area, hence partially disrupting the dmp-tryptophan $\pi$-stacking arrangement but maintain a dmp-tryptophan proximity (ca. $8 \AA$ in the first $150 \mathrm{~ns}$ of simulation). This feature can be understood in terms of the electronic density reorganization in the triplet state leading to a positive charge accumulation in the carbonyl region and hence to a favorable electrostatic interaction with $\mathrm{dmp}$. On the other hand, in the case of the ${ }^{3} \mathrm{CS}$ state the positively and negatively charged $\mathrm{dmp}$ and tryptophan strongly interact together, with dominant electrostatic interaction over dispersion and leading to a partial loss of the $\pi$-stacking conformation. Also in this case, $\mathrm{dmp}$ and tryptophan side chain remain close, with a distance between 7 and $8 \AA$ when a stable conformation is found (mainly after $100 \mathrm{~ns}$ ).

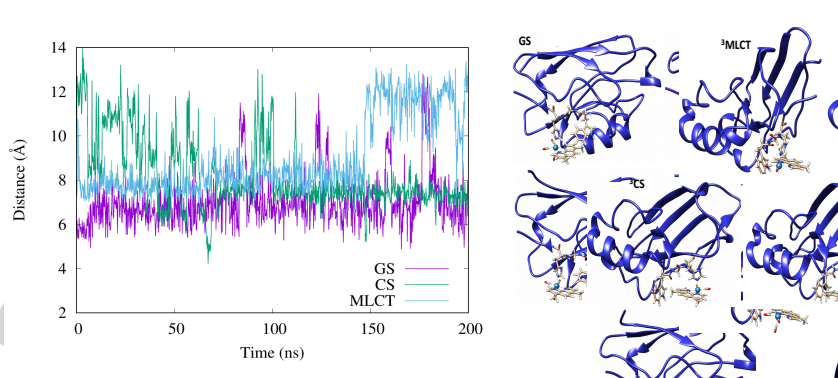

Figure 5. Time evolution of the dmp-tryptophan distance in the azurin protein and three representative conformations of the GS, ${ }^{3} \mathrm{MLCT}$, and ${ }^{3} \mathrm{CS}$ state for $\left[\operatorname{Re}^{\prime}(\mathrm{dmp})(\mathrm{CO})_{3}(\mathrm{His} 124)(\operatorname{Trp} 122)\right]$ as extracted from the respective MD trajectory.

However, even if the stacking is partially loss in the excited triplet state manifold, the role of the protein environment in constraining the conformation and the close proximity of the chromophore units is evident. Furthermore, it has to be noted that our MD simulations have been ran for 200 ns even if the longest triplet lifetime is of the order of some tenths of ns. This intentional choice was taken to allow for a better sampling of the conformational space. However the disruption of the stacking and a steep increase in the dmp-tryptophan distance, as observed at the end of the ${ }^{3} \mathrm{MLCT}$ simulation, appears as overestimated. Indeed, in the photophysical process the system will most likely span regions of space closer to the GS conformation, i.e. evolve in a relatively well stacked environment.

Characterization of the low-lying ${ }^{3} \mathrm{MLCT}$ and ${ }^{3} \mathrm{CS}$ excited states of $\left[\operatorname{Re}^{\prime}(d m p)(C O)_{3}(H i s 124)(\operatorname{Trp122})\right]$ in water and in the protein

The absorption spectrum in the protein environments was calculated at QM/MM level and are presented in Figure 6 also compared with the one obtained in water. An analysis of the convergence of the simulated absorption spectrum with the number of snapshots is presented in Supplementary Information (Figure S1). One can immediately note that its main characteristic, such as the low lying ${ }^{1} \mathrm{MLCT}$ band, describing a transition from $\operatorname{Re}(\mathrm{CO})_{3}$ to $\mathrm{dmp}$, is present when considering both environments. Nevertheless, a red shift of ca. $90 \mathrm{~nm}$ is observed from water $(360 \mathrm{~nm})$ to protein $(450 \mathrm{~nm})$, most probably due to the modification in the electrostatic surroundings. In any case, excitation to the ${ }^{1} \mathrm{MLCT}$ is also confirmed by the application of $400 \mathrm{~nm}$ laser-pulses in previous experiments [20], followed by intersystem crossing to populate the relevant ${ }^{3} \mathrm{MLCT}$ state. 
At higher energies (ca. $290 \mathrm{~nm}$ ) a second peak is found in the protein, corresponding to a ${ }^{1} \mathrm{CS}$ state and thus involving tryptophan side chain and dmp. This transition can be found also in water (see Table S1 in Supporting Information) but is mainly hided by the largely unstructured shape of the low lying ${ }^{1} \mathrm{MLCT}$ band, suggesting once more that the high flexibility shown by the complex in water hampers the possibility to correctly describe the real system. At even higher energies the most intense peak is found to be due to the sum of different types of transition, mainly MLCT to the histidine side chain, inter-ligand (IL) and partially ligand-to-ligand charge transfer (LLCT), as expected for such complexes in the UVB region.

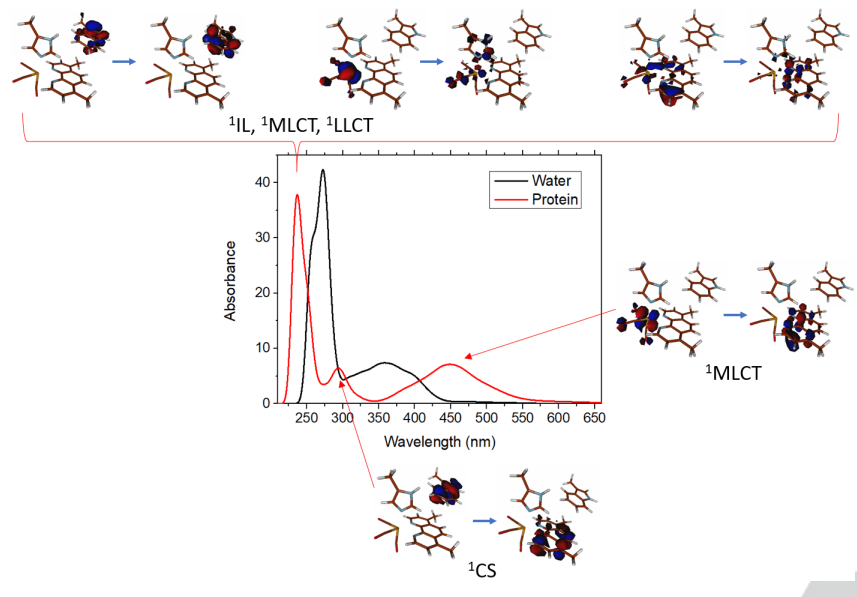

Figure 6. Calculated absorption spectrum as a Gaussian convolution ( $F W H M=0.2 \mathrm{eV}$ ) from 100 snapshots along the GS dynamics, considering both water (black) and protein (red) environments. Moreover, occupied (left) and virtual (right) natural transition orbitals are shown, for each peak of the protein spectrum.

On the other hand, the situation is significantly different when considering the behavior and in particular the spin density distribution of the MLCT and CS triplet states. Indeed, it should be recalled that in the case of the isolated system a geometrical constraint is needed to avoid the collapse of the ${ }^{3} \mathrm{MLCT}$ state on the ${ }^{3} \mathrm{CS}$ during the geometry optimization. This behavior was rather puzzling since the timescale reported from the pump probe experiments definitively points to the existence of stable ${ }^{3} \mathrm{MLCT}$ and ${ }^{3} \mathrm{CS}$ minima whose interconversion happens in the ns regime.

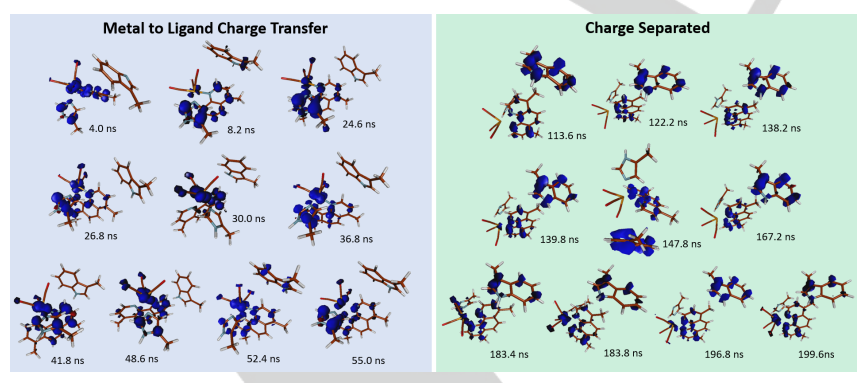

Figure 7. QM/MM spin densities obtained for the ${ }^{3} \mathrm{MLCT}$ and ${ }^{3} \mathrm{CS}$ state, respectively, after $\mathrm{QM} / \mathrm{MM}$ optimization of selected MD snapshots, when including the whole azurin protein as environment.
As reported in the Supplementary Information, we note that for both trajectories QM/MM single-point calculations of selected snapshots are able to retrieve the correct electronic nature and in particular the spin distribution of the two states (Figure S2). Hence, in the case of the ${ }^{3} \mathrm{MLCT}$ the unpaired electrons are concentrated on the $\operatorname{Re}(\mathrm{CO})_{3}$ and on the dmp unit, while for ${ }^{3} \mathrm{CS}$ the spin density peaks on the tryptophan and on dmp. Most importantly, as reported in Figure 7, such spin density pattern is also maintained upon geometry optimization. As a consequence, we may conclude that the environmental effects provided by the protein, either electrostatic or via geometric constraints, allows for the stabilization of the ${ }^{3} \mathrm{MLCT}$ state and the formation of a relative minimum that may be correlated to the metastable specie observed experimentally with a lifetime in the nanosecond domain. From a more methodological point of view, those results are also confirming the soundness of our strategy of FF parameterization, allowing the description of the time evolution of the triplet excited states of organometallic compounds.

\section{Conclusions}

We have performed a truthful multiscale characterization of the ground state and excited state behaviors of $\left[\operatorname{Re}^{\prime}(\mathrm{dmp})(\mathrm{CO})_{3}(\mathrm{His} 124)(\operatorname{Trp} 122)\right]$ both in solution and embedded in an azurin protein. In particular, we exploited a combination of classic MD to sample the conformational space, with high-level quantum chemistry to obtain the relevant electronic structure properties. On this context we also present a strategy allowing the parameterization of force field for electronic excited triplet states in particular taking into account the different charge distribution.

We have evidenced that, differently from the situation in the azurin environment, the GS of the solvated $\left[\operatorname{Re}^{\prime}(\mathrm{dmp})(\mathrm{CO})_{3}(\right.$ His 124)(Trp122)] exhibits a high flexibility, inducing an important distance between $\mathrm{dmp}$ and tryptophan units. Direct consequence of this flexibility is the strong decrease in the coupling between the chromophores, and hence in the hampering of the charge transfer state mechanisms. This explains why, in the case of the solvated model system, timeresolved spectroscopy has not evidenced any charge-transfer process. This situation is totally reversed when the system is embedded in a protein matrix. Indeed in this case the coupling between the two units is globally maintained, allowing for the transfer to take place. More interestingly performing QM/MM calculations on top of snapshots extracted from classic MD describing the ${ }^{3} \mathrm{MLCT}$ and ${ }^{3} \mathrm{CS}$ states, respectively, we have shown that the two states represent local minima in the potential energy surface and may hence give rise to metastable species. This aspect is in contrast with the situation observed for the isolated model system in which QM optimization was leading to the collapse on the ${ }^{3} \mathrm{CS}$ state, hence not enabling to describe the ${ }^{3} \mathrm{MLCT}$ state. The existence of metastable triplet states separated by a non-negligible energy barrier is indeed necessary to explain the ns time component observed experimentally for the formation of the ${ }^{3} \mathrm{CS}$ state.

Our results provide an important rationalization of the role of the azurin protein in tuning the evolution of the excited states potential and therefore the photophysical properties of organometallic processes. On the other hand, this work constitutes a fundamental preliminary step allowing the correct sampling of the different potential energy transfer involved in the 
global process. In the future we will provide the full characterization of the experimentally observed photo-process including, on one side, the use of non-adiabatic moleculardynamics to unravel the short time-scale and, on the other side, the performance of biased QM/MM dynamics to explore the free energy potential related to the slower ${ }^{3} \mathrm{MLCT} /{ }^{3} \mathrm{CS}$ interconversion.

\section{Computational Methodology}

Electronic structure of solvated [Re'(dmp)(CO) $\left.{ }_{3}(H i s 124)(\operatorname{Trp122})\right]$

Quantum Chemistry ground state calculations were performed by means of density functional theory (DFT) including solvent corrections based on COSMO [16-18] with dielectric constant $\varepsilon=78.39$ for water. The

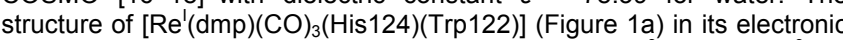
singlet ground state and lowest triplet excited states, ${ }^{3} \mathrm{MLCT}$ and ${ }^{3} \mathrm{CS}$ has been optimized with DFT and UDFT, respectively. In all cases the initial nuclear coordinates were extracted from the X-ray crystal structure reported in ref [24]. Convergence of the ${ }^{3} \mathrm{MLCT}$ state has been achieved under geometrical constraint, namely by freezing partly the structure of the (His124)(Trp122) fragment in order to avoid convergence to the ${ }^{3} \mathrm{CS}$ state. The calculations were performed using the B3LYP functional [28] including Grimme D3 dispersion corrections [29], and an all-electron triple- $\xi$ basis set [30]. The scalar relativistic effects were taken into account within the zeroth-order regular approximation (ZORA) [31]. The low-lying excited states have been computed by means of timedependent DFT (TD-DFT) using the B3LYP functional. These calculations were performed with the ADF2013 code [32,33].

\section{FF parameterizations}

Table 1. Charges of the molecular fragments used to parameterize the FF for the different electronic states..

\begin{tabular}{lcccc}
\hline State & $\operatorname{Re}(\mathrm{CO})_{3}$ & $\mathrm{dmp}$ & $\operatorname{Trp}$ & $\mathrm{Cu}$ \\
\hline $\mathrm{GS}$ & +1 & 0 & 0 & +1 \\
${ }^{3} \mathrm{MLCT}$ & +2 & -1 & 0 & +1 \\
${ }^{3} \mathrm{MC}$ & +1 & -1 & +1 & +1 \\
\hline
\end{tabular}

The starting geometries used for the development of new FF parameters were the equilibrium structures of GS, MLCT and CS states obtained after QM optimization on the model system made up of the rhenium complex, the tryptophan side chain and a bridging glycine residue. The dangling $\mathrm{C}=\mathrm{O}$ and $\mathrm{N}-\mathrm{H}$ termini groups were capped by their respective terminal amide groups. Atomic point charges of the model system have been computed through a fit of the QM electrostatic potential, based on the conventional restrained electrostatic potentia (RESP) [34] procedure at the DFT level of theory. Especially, the B3LYP functional was employed together with the $6-31 \mathrm{G}^{*}$ basis set for $\mathrm{O}, \mathrm{N}, \mathrm{C}$ and $\mathrm{H}$ atoms, and the LANL2DZ basis set for the rhenium atom (with a van der Waals radii set to $1.6 \AA$ ). In order to match the required formal charges for each residue in each case, a fragment approach was used. In particular for ${ }^{3} \mathrm{MLCT}$, in which the electron density is drawn from the metal to the dmp ligand, charges were obtained considering a -1 charged dmp moiety, while a +1 positive charge was redistributed equally to each carbonyl $\mathrm{CO}$ and the Re atom. This choice is justified by the fact that $\mathrm{Re}$ and $\mathrm{CO}$ ligands are strongly correlated with hole density spread over those units in both triplet and single MLCT states [10]. In the case of CS state instead, a positively charged tryptophan moiety was considered together with the negatively charged $\mathrm{dmp}$. The following procedure was applied: $i$ ) the charges of the glycine residue as well as the lateral $\mathrm{CH}_{2}$ chain bridging the aminoacid to the rhenium complex and the tryptophan were set to the canonical point charges from the Amber force field [35]; ii) the missing charge generated by the first step is equally redistributed over each other atom to obtain an exact total charge of +1 ; iii) based on the experimentally determined charge distribution for each case (see Table 1) the atomic point charges of each subunit (rhenium, dmp or tryptophan) have been separately shifted to obtain the correct final charges corresponding to either GS, MLCT or CS state, with a global charge always equal to +1 . The force field bonded and non-bonded parameters of the Rhenium complex have been taken from previous work [10], and those of the tryptophan and glycine where taken from the AmberFF14 force field for amino acids [36]. Moreover, since the point charges and FF parameters of the backbone were directly taken from the AmberFF14 force field, the inclusion of the model system (without capping groups) in the azurin protein has been done without the need of any further modification or tuning. Only a redistribution of the atomic point charges of the capping residues in the model system over all other atoms has been performed.

Furthermore, the mutant azurin is a native metalloprotein containing a copper atom bound to a cysteine residue and complexed with two histidine residues, a sulphur atom of a methionine residue and the oxygen atom of a close-by peptide bond. For this system the force field parameters have been taken from reference [37]. In this case the copper atom has been considered as bound to the cysteine with an atomic point charge set to +1

$M D$ simulations

The starting structure of each MD simulation (GS, MLCT and CS for model system and protein) was placed in a truncated octahedron containing a $10 \AA$ buffer of water molecules as well as 1 (model system) or 2 (protein) $\mathrm{Na}^{+}$ions to neutralize the global charge of the system. Periodic boundary conditions were employed as well as Particle Mesh Ewald for Coulomb interactions and the Shake algorithm to reduce the degrees of freedom of hydrogen atoms. Each simulation started with 4000 steps of energy minimization using the steepest descent algorithm followed by 4000 using conjugated gradient. Then a thermalization step has been performed in the NVT ensemble for $200 \mathrm{fs}$ bringing the temperature from 0 to $300 \mathrm{~K}$. This was followed by $400 \mathrm{fs}$ of equilibration in the NPT ensemble to relax the density of the entire system and finally 200 ns of production runs (NPT ensemble) were performed. The temperature $(300 \mathrm{~K})$ and pressure $(1 \mathrm{~atm})$ were kept constant using the Monte Carlo thermostat and Langevin dynamics. All MD simulations were performed using the Amber suite of codes [38].

\section{QM/MM calculations}

All the QM/MM calculations have been performed on an ensemble of 100 snapshots extracted from each GS MD trajectory of the Re complex in water and including the azurin protein, while 10 snapshots were taken from the ${ }^{3} \mathrm{MLCT}$ and ${ }^{3} \mathrm{CS}$ MD trajectories. In all cases, the snapshots were chosen to represent the conformational space phase sampled by the respective MD trajectory. In all the cases the $\mathrm{QM}$ partition consisted of the Re atoms, the three CO units, dmp, the imidazole ring, and finally the coupled tryptophan side chain. All the other protein residues, including the bridging glycine, were treated at MM level as well as solvent molecules and the neutralizing ions. QM/MM calculations were performed at DFT level, with the unrestricted formalism in the case of the triplet states, using the B3LYP functional corrected with the D3 scheme of Grimme to include dispersion effects. A LANL2DZ basis set including pseudopotential for the Re atom was used throughout the calculation. From the GS snapshots, the absorption spectrum was calculated using TD-DFT formalism as a convolution of all the vertical transitions obtained for each snapshot, following a strategy currently used in our groups [10,39-44].

For each of the snapshots obtained from the ${ }^{3} \mathrm{MLCT}$ and ${ }^{3} \mathrm{MC}$ dynamics the lowest triplet state $\left(\mathrm{T}_{1}\right)$ was calculated at DFT level and the spin density analysed to assure the stability of the electronic state. Finally a QM/MM geometry optimization of every snapshot was performed, calculating again the spin density. All the QM/MM calculations were carried out using the Terachem code [45] with its interface with Amber suite to treat the MM environment at electrostatic embedding level [46]. The Pulay's hybrid DIIS/A-DIIS algorithm [47] together with an increase threshold on the two electron integrals of $10^{-15}$ was used to avoid SCF 
convergence failure. Excited state nature has also been characterized in terms of natural transition orbitals (NTOs) $[48,49]$

\section{Acknowledgements}

This work is supported by the French/Austrian ANR-15-CE290027-01 DeNeTheor, the European actions COST perspect- $\mathrm{H}_{2} \mathrm{O}$, CODEC, and ECOST-Bio. The Labex "Chimie des Systèmes Complexes" (ANR-10-LABX-0026_CSC) is acknowledged. The quantum chemical calculations were performed on the computer nodes of the LCQS, Strasbourg, and thanks to the computer facilities of the High Performance Computing regional center of Univ. of Strasbourg. M. M. acknowledges the University of La Rioja for a postdoctoral contract.

Keywords: Electron transfer $•$ Metal Sensitized Proteins • Environmental Effects $• \mathrm{QM} / \mathrm{MM} \cdot$ Charge Separated States

\section{References}

1. Sánchez-Murcia, P.A., Nogueira, J.J., and González, L. (2018) Exciton Localization on Ru-Based Photosensitizers Induced by Binding to Lipid Membranes. J. Phys. Chem. Lett., 9 (4), 683-688. Atkins, A.J., and González, L. (2017) Trajectory Surface-Hopping Dynamics Including Intersystem Crossing in [Ru(bpy)3]2+. J. Phys. Chem. Lett., 8 (16), 3840-3845.

3. Gourlaouen, C., Eng, J., Otsuka, M., Gindensperger, E., and Daniel, C. (2015) Quantum Chemical Interpretation of Ultrafast Luminescence Decay and Intersystem Crossings in Rhenium(I) Carbonyl Bipyridine Complexes. J. Chem. Theory Comput., 11 (1), 99-110.

4. Eng, J., Gourlaouen, C., Gindensperger, E., and Daniel, C. (2015) Spin-Vibronic Quantum Dynamics for Ultrafast Excited-State Processes. Acc. Chem. Res., 48 (3), 809-817. Daniel, C. (2015) Absorption spectroscopy, emissive properties, and ultrafast intersystem crossing processes in transition metal complexes: TD-DFT and spin-Orbit coupling. Top. Curr. Chem., 368, 377-414.

6. Daniel, C. (2015) Photochemistry and photophysics of transition metal complexes: Quantum chemistry. Coord. Chem. Rev., 282283, 19-32.

7. Chantzis, A., Very, T., Daniel, C., Monari, A., and Assfeld, X. (2013) Theoretical evidence of photo-induced charge transfer from DNA to intercalated ruthenium (II) organometallic complexes. Chem. Phys. Lett., 578. Chantzis, A., Very, T., Monari, A., and Assfeld, X. (2012) Improved Treatment of Surrounding Effects: UV/vis Absorption Properties of a Solvated Ru(II) Complex. J. Chem. Theory Comput., 8 (5), 15361541.

9. Véry, T., Ambrosek, D., Otsuka, M., Gourlaouen, C., Assfeld, X. Monari, A., and Daniel, C. (2014) Photophysical properties of ruthenium(ii) polypyridyl dna intercalators: Effects of the molecular surroundings investigated by theory. Chem. - A Eur. J., 20 (40), 12901-12909.

Mai, S., Gattuso, H., Fumanal, M., Muñoz-Losa, A., Monari, A., Daniel, C., and González, L. (2017) Excited-states of a rhenium carbonyl diimine complex: Solvation models, spin-orbit coupling, and vibrational sampling effects. Phys. Chem. Chem. Phys., 19 (40).

11. Ambrosek, D., Loos, P.F., Assfeld, X., and Daniel, C. (2010) A theoretical study of $\mathrm{Ru}$ (II) polypyridyl DNA intercalators. Structure and electronic absorption spectroscopy of [Ru(phen)2(dppz)]2+and $[R u(\operatorname{tap}) 2(\mathrm{dppz})] 2+$ complexes intercalated in guanine-cytosine base pairs. J. Inorg. Biochem., 104 (9), 893-901.

12. Cossi, M., Barone, V., Cammi, R., and Tomasi, J. (1996) Ab initio study of solvated molecules: A new implementation of the polarizable continuum model. Chem. Phys. Lett., 255 (4-6), 327335.

13. Cancès, E., Mennucci, B., and Tomasi, J. (1997) A new integral equation formalism for the polarizable continuum model: Theoretical background and applications to Isotropic and anisotropic dielectrics. J. Chem. Phys., 107 (8), 3032-3041.

14. Barone, V., and Cossi, M. (1998) Quantum calculation of molecular energies and energy gradients in solution by a conductor solvent model. J. Phys. Chem. A, 102 (11), 1995-2001. Mennucci, B. (2012) Polarizable continuum model. Wiley Interdiscip. Rev. Comput. Mol. Sci., 2 (3), 386-404.

Klamt, A., and Schüürmann, G. (1993) COSMO: a new approach to dielectric screening in solvents with explicit expressions for the screening energy and its gradient. J. Chem. Soc., Perkin Trans. 2, (5), 799-805.

Klamt, A. (1995) Conductor-like screening model for real solvents: A new approach to the quantitative calculation of solvation phenomena. J. Phys. Chem., 99 (7), 2224-2235. Klamt, A., and Jonas, V. (1996) Treatment of the outlying charge in continuum solvation models. J. Chem. Phys., 105 (22), 9972-9981. Blanco-Rodríguez, A.M., Di Bilio, A.J., Shih, C., Museth, A.K., Clark, I.P., Towrie, M., Cannizzo, A., Sudhamsu, J., Crane, B.R., Sýkora, J., Winkler, J.R., Gray, H.B., Záliš, S., and Vlček, A. (2011) Phototriggering electron flow through Rel-modified pseudomonas aeruginosa azurins. Chem. - A Eur. J., 17 (19), 5350-5361. Blanco-Rodríguez, A.M., Busby, M., Gradinaru, C., Crane, B.R., Di Bilio, A.J., Matousek, P., Towrie, M., Leigh, B.S., Richards, J.H., Vlcek, A., and Gray, H.B. (2006) Excited-state dynamics of structurally characterized $[\operatorname{Rel}(\mathrm{CO}) 3($ phen $)(\mathrm{His} X)]+(X=83,109)$ Pseudomonas aeruginosa azurins in aqueous solution. J. Am. Chem. Soc., 128 (13), 4365-70.

21. Blanco-Rodríguez, A.M., Busby, M., Ronayne, K., Towrie, M., Grădinaru, C., Sudhamsu, J., Sýkora, J., Hof, M., Záliš, S., Di Bilio, A.J., Crane, B.R., Gray, H.B., and Vlček, A. (2009) Relaxation dynamics of Pseudomonas aeruginosa $\operatorname{Rel}(\mathrm{CO}) 3($ a-diimine)(HisX)+ $(X=83,107,109,124,126)$ Cull azurins. J. Am. Chem. Soc., 131 (33), 11788-11800.

22. Véry, T., Ambrosek, D., Otsuka, M., Gourlaouen, C., Assfeld, X., Monari, A., and Daniel, C. (2014) Photophysical properties of ruthenium(ii) polypyridyl dna intercalators: Effects of the molecular surroundings investigated by theory. Chem. - A Eur. J., 20 (40). 
Chantzis, A., Very, T., Despax, S., Issenhuth, J.-T., Boeglin, A., Hébraud, P., Pfeffer, M., Monari, A., and Assfeld, X. (2014) UV-vis absorption spectrum of a novel Ru(II) complex intercalated in DNA: $\left[\mathrm{Ru}\left(2,2^{\prime} \text {-bipy)(dppz)(2,2'-ArPy) }\right]^{+}\right.$. J. Mol. Model., 20 (3). Shih, C., Museth, A.K., Abrahamsson, M., Blanco-Rodriguez, A.M., Di Bilio, A.J., Sudhamsu, J., Crane, B.R., Ronayne, K.L., Towrie, M. Vlček, A., Richards, J.H., Winkler, J.R., and Gray, H.B. (2008) Tryptophan-accelerated electron flow through proteins. Science (80. )., 320 (5884), 1760-1762.

Monari, A., Rivail, J.L., and Assfeld, X. (2013) Theoretical modeling of large molecular systems. Advances in the local self consistent field method for mixed quantum mechanics/molecular mechanics calculations. Acc. Chem. Res., 46 (2), 596-603. Adamo, C., and Barone, V. (1999) Toward reliable density functional methods without adjustable parameters: The PBE0 model. J. Chem. Phys., 110, 6158-6169. Zhao, Y., and Truhlar, D.G.J. (2008) The M06 suite of density functionals for main group thermochemistry, thermochemical kinetics, noncovalent interactions, excited states, and transition elements: two new functionals and systematic testing of four M06class functionals and 12 other function. Theor. Chem. Acc., 120 (1), 215-241. Becke, A. (1993) B3LYP. J. Chem. Phys., 98, 5648-5652. Grimme, S., Antony, J., Ehrlich, S., and Krieg, H. (2010) A consistent and accurate ab initio parametrization of density functional dispersion correction (DFT-D) for the 94 elements H-Pu. J. Chem. Phys., 132 (15), 154104. Van Lenthe, E., and Baerends, E.J. (2003) Optimized Slater-type basis sets for the elements 1-118. J. Comput. Chem., 24 (9), 11421156.

31. van Lenthe, E., Baerends, E.J., and Snijders, J.G. (1994) Relativistic total energy using regular approximations. J. Chem. Phys., 101 (11), 9783-9792. Baerends, E.J., Ziegler, T., Atkins, A.J., Autschbach, J., Bashford, D., Baseggio, O., Bérces, A., Bickelhaupt, F.M., Bo, C., Boerritger, P.M., Cavallo, L., Daul, C., Chong, D.P., Chulhai, D. V, Deng, L., Dickson, R.M., Dieterich, J.M., Ellis, D.E., van Faassen, M., Ghysels, A., Giammona, A., van Gisbergen, S.J.A., Goez, A., Götz, A.W., Gusarov, S., Harris, F.E., van den Hoek, P., Hu, Z., Jacob, C.R., Jacobsen, H., Jensen, L., Joubert, L., Kaminski, J.W., van Kessel, G., König, C., Kootstra, F., Kovalenko, A., Krykunov, M., van Lenthe, E., McCormack, D.A., Michalak, A., Mitoraj, M., Morton, S.M., Neugebauer, J., Nicu, V.P., Noodleman, L., Osinga, V.P., Patchkovskii, S., Pavanello, M., Peeples, C.A., Philipsen, P.H.T., Post, D., Pye, C.C., Ramanantoanina, H., Ramos, P., Ravenek, W., Rodríguez, J.I., Ros, P., Rüger, R., Schipper, P.R.T., Schlüns, D., van Schoot, H., Schreckenbach, G., Seldenthuis, J.S., Seth, M., Snijders, J.G., Solà, M., M., S., Swart, M., Swerhone, D., te Velde, G., Tognetti, V., Vernooijs, P., Versluis, L., Visscher, L., Visser, O. Wang, F., Wesolowski, T.A., van Wezenbeek, E.M., Wiesenekker, G., Wolff, S.K., Woo, T.K., and Yakovlev, A.L. ADF2017, SCM, Theoretical Chemistry, Vrije Universiteit, Amsterdam, The Netherlands, https://www.scm.com. van Gisbergen, S.J.A., Snijders, J.G., and Ziegler, T. (2001) Chemistry with ADF. J. Comput. Chem., 22 (9), 931-967.

34. Wang, J., Cieplak, P., and Kollman, P.A. (2000) How well does a restrained electrostatic potential (RESP) model perform in calculating conformational energies of organic and biological molecules? J. Comput. Chem., 21 (12), 1049-1074. Wang, J., Wolf, R.M., Caldwell, J.W., Kollman, P.A., and Case, D.A. (2004) Development and testing of a general Amber force field. J. Comput. Chem., 25 (9), 1157-1174.

36. Maier, J.A., Martinez, C., Kasavajhala, K., Wickstrom, L., Hauser, K.E., and Simmerling, C. (2015) ff14SB: Improving the Accuracy of Protein Side Chain and Backbone Parameters from ff99SB. J. Chem. Theory Comput., 11 (8), 3696-3713.

37. Monari, A., Very, T., Rivail, J.L., and Assfeld, X. (2012) Effects of mutations on the absorption spectra of copper proteins: A QM/MM study. Theor. Chem. Acc., 131 (5), 1-9.

38. Case, D.A., Berryman, J.T., Betz, R.M., Cerutti, D.S., T. E. Cheatham, I.I.I., Darden, T.A., Duke, R.E., Giese, T.J., Gohlke, H., Goetz, A.W., Homeyer, N., Izadi, S., Janowski, P., Kaus, J., Kovalenko, A., Lee, T.S., Grand, S. Le, Luchko, P.L.T., Luo, R., B. Madej a, d K.M.M., Monard, G., Needham, P., Nguyen, H.T., Nguyen, H.T., Omelyan, I., Onufriev, A., Roe, D.R., Roitberg, A., Salomon-Ferrer, R., Simmerling, C.L., Smith, W., Swails, J., Walker, R.C., Wang, J., Wolf, R.M., Wu, X., York, D.M., Kollman, P.A., CheathamIII, T.E., Darden, T.A., Duke, R.E., Giese, T.J., Gohlke, H., Goetz, A.W., Homeyer, N., Izadi, S., Janowski, P., Kaus, J., Kovalenko, A., Lee, T.S., LeGrand, S., Li, P., Luchko, T., Luo, R., Madej, B., Merz, K.M., Monard, G., Needham, P., Nguyen, H.T., Nguyen, H.T., Omelyan, I., Onufriev, A., Roe, D.R., Roitberg, A., Salomon-Ferrer, R., Simmerling, C.L., Smith, W., Swails, J., Walker, R.C., Wang, J., Wolf, R.M., Wu, X., York, D.M., Kollman, P.A., and et al., D.A.C. (2015) AMBER 2015. AMBER 15, Universiyt Calif. San Fr.

Etienne, T., Gattuso, H., Monari, A., and Assfeld, X. (2014) QM/MM modeling of Harmane cation fluorescence spectrum in water solution and interacting with DNA. Comput. Theor. Chem., 10401041.

40. Gattuso, H., Marazzi, M., Dehez, F., and Monari, A. (2017) Deciphering the photosensitization mechanisms of hypericin towards biological membranes. Phys. Chem. Chem. Phys., 19 (34). Gattuso, H., Besancenot, V., Grandemange, S., Marazzi, M., and Monari, A. (2016) From non-covalent binding to irreversible DNA lesions: Nile blue and nile red as photosensitizing agents. Sci. Rep., 6, 28480 .

42. Marazzi, M., Gattuso, H., and Monari, A. (2016) Nile blue and Nile red optical properties predicted by TD-DFT and CASPT2 methods: static and dynamic solvent effects. Theor. Chem. Acc., 135 (3), 111.

43. Gattuso, H., Dumont, E., Marazzi, M., and Monari, A. (2016) Twophoton-absorption DNA sensitization: Via solvated electron production: Unraveling photochemical pathways by molecular modeling and simulation. Phys. Chem. Chem. Phys., 18 (27).

44. Marazzi, M., Gattuso, H., Monari, A., and Assfeld, X. (2018) Steadystate linear and non-linear optical spectroscopy of organic 
chromophores and bio-macromolecules. Front. Chem., 6 (APR)

45. Titov, A. V., Ufimtsev, I.S., Luehr, N., and Martinez, T.J. (2013) Generating efficient quantum chemistry codes for novel architectures. J. Chem. Theory Comput., 9 (1), 213-221.

46. Götz, A.W., Clark, M.A., and Walker, R.C. (2014) An extensible interface for QM/MM molecular dynamics simulations with AMBER. J. Comput. Chem., 35 (2), 95-108.

47. Pulay, P. (1982) Improved SCF convergence acceleration. J. Comput. Chem., 3 (4), 556-560.
48.

Martin, R.L. (2003) Natural transition orbitals. J. Chem. Phys., 118 (11), 4775-4777

49. Etienne, T., Assfeld, X., and Monari, A. (2014) Toward a quantitative assessment of electronic transitions" charge-transfer character. J. Chem. Theory Comput., 10 (9), 3896-3905. 


\section{Entry for the Table of Contents}

Layout 1:

\section{FULL PAPER}

We present the molecular modeling and simulation of the lowest excited states of a $\mathrm{Re}$ sensitized azurine. We evidence the crucial role of the environment to allow the stabilization of charge separated states and hence providing a path to longrange electron transfer.

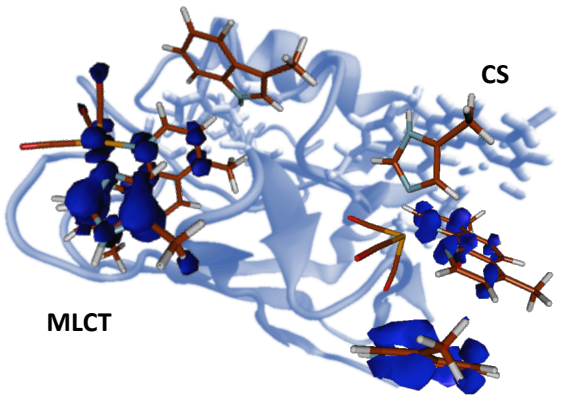

M. Marazzi, H. Gattuso, M. Fumanal, C. Daniel", and A. Monari*

Page No. - Page No.

Charge transfer vs. charge separated triplet excited states of $\left[\operatorname{Re}^{\prime}(\mathrm{dmp})(\mathrm{CO})_{3}(\mathrm{His} 124)(\operatorname{Trp} 122)\right]$ in water and in modified pseudomonas aeruginosa azurin protein. 